\title{
Comparison of the clinical features and therapeutics of COVID- 19 in cardio-cerebrovascular disease (CCVD) and non-CCVD patients
}

\author{
Yu Wang ${ }^{1, *}$, Lan $\mathrm{Li}^{1, *}$, Yuanjiang $\mathrm{Pan}^{2}, \mathrm{Yu} \mathrm{He}^{3}$, Zuhua $\mathrm{Chen}^{5}$, Yunhao Xun ${ }^{5}$, Yuhan $\mathrm{Xu}^{1}$, Yilei Guo ${ }^{1}$, Jiehong Yang $(\bowtie)^{4}$, \\ Jianchun Guo $(\bowtie)^{5}$, Haitong Wan $(\bowtie)^{1}$ \\ ${ }^{1}$ Institute of Cardio-cerebrovascular Disease, Zhejiang Chinese Medical University, Hangzhou 310053, China; ${ }^{2}$ Department of Chemistry, \\ Zhejiang University, Hangzhou 310027, China; ${ }^{3}$ College of Pharmaceutical Science, Zhejiang Chinese Medical University, Hangzhou \\ 310053, China; ${ }^{4}$ School of Basic Medical Sciences and Public Health, Zhejiang Chinese Medical University, Hangzhou 310053, China; \\ ${ }^{5}$ Integrated TCM \& Western Medicine Department, Xixi Hospital of Hangzhou, Hangzhou 310023, China
}

(C) Higher Education Press 2021

\begin{abstract}
Cardio-cerebrovascular disease (CCVD) is a major comorbidity of coronavirus disease 2019 (COVID19). However, the clinical characteristics and outcomes remain unclear. In this study, 102 cases of COVID-19 from January 22, 2020 to March 26, 2020 in Xixi Hospital of Hangzhou were included. Twenty cases had pre-existing CCVD. Results showed that compared with non-CCVD patients, those with CCVD are more likely to develop severe disease (15\% versus 1\%), and the proportion of pneumonia severity index grade IV was significantly higher (25\% versus 3.6\%). Computed tomography images demonstrated that the proportion of multiple lobe lesion involvement was significantly higher in the CCVD group than in the non-CCVD group (90\% versus $63.4 \%$ ). Compared with non-CCVD group, the levels of C-reactive protein, fibrinogen, D-dimer, and serum amyloid-A were higher, whereas the total protein and arterial partial $\mathrm{PaO}_{2}$ were lower in the CCVD group. Although no statistical difference was observed in the outcomes between groups, CCVD patients received more intensive comprehensive treatment to improve COVID-19 symptoms compared with non-CCVD patients. Integrated Chinese and Western medicine treatments have certain advantages in controlling the severe conversion rate and mortality of COVID-19. In addition, given that COVID-19 patients are usually related to coagulation disorders and thrombosis risk, the application of Chinese medicine in promoting blood circulation and removing stasis should be strengthened.
\end{abstract}

Keywords COVID-19; cardio-cerebrovascular disease; traditional Chinese medicine; clinical features; clinical therapeutics

\section{Introduction}

Severe acute respiratory syndrome coronavirus 2 (SARSCoV-2) is a newly emerging, highly contagious coronavirus [1]; it mainly invades the respiratory tract and lungs, leading to a new type of coronavirus pneumonia [2]. On December 31, 2019, the first case of severe pneumonia in

Received May 28, 2020; accepted September 16, 2020

Correspondence: Jiehong Yang, yjhong@zcmu.edu.cn; Jianchun Guo, guojianchun1961@126.com; Haitong Wan, haitongw@163.com

${ }^{*}$ Yu Wang and Lan Li contributed equally to this work. humans was reported [3]. In February 2020, the World Health Organization (WHO) designated this case as coronavirus disease 2019 (COVID-19) [4]. On March 11, 2020, the WHO declared COVID-19 as a public health emergency of international concern [5]. According to the data received by the WHO from national authorities at 10:00 CEST on June 29, 2020, the COVID-19 outbreak has infected 10021401 individuals and caused 499913 deaths (approximately 4.99\% mortality) globally [6]. The transmission routes of COVID-19 include direct contact, droplet, and possible aerosol transmission [7]. Populations are generally susceptible to COVID-19. Moreover, individuals with hypertension, cardiovascular disease, and cerebrovascular disease are more susceptible to severe or 
critical disease and often admitted to the intensive care unit (ICU) [8]. Zhou et al. [9] investigated 191 cases of COVID-19 in Wuhan and observed that the elderly with comorbidities of hypertension (30\%), coronary heart disease (8\%), and diabetes (19\%) are prone to develop acute respiratory distress syndrome. Chen et al. [10] collected data from 249 laboratory confirmed cases and reported cardio-cerebrovascular disease (CCVD) as the most common comorbidity (21.7\%). However, the clinical characteristics and outcomes of patients with COVID-19 and CCVD remain unclear.

This study aimed to compare the clinical features of COVID-19 in CCVD and non-CCVD patients and summarize the effects of integrated Chinese and Western medicine on the treatment of COVID-19.

\section{Methods}

\section{Study design and participants}

In this single-center retrospective study, we recruited patients at Xixi Hospital of Hangzhou (the designated COVID-19 treatment hospital in Hangzhou, China) from January 22, 2020 to March 26, 2020. Based on the presence or absence of CCVD in COVID-19 patients before admission, the patients were divided into the CCVD and non-CCVD groups. CCVD was defined as hypertension, coronary heart disease, angina pectoris, myocardial infarction, cerebral infarction, cerebral hemorrhage, ischemic stroke, and hyperlipidemia. The study was approved by the Ethics Committee of Xixi Hospital of Hangzhou (Approval No.: 2020-14).

\section{Diagnosis criteria}

In accordance with the Chinese national guidelines for COVID-19 diagnosis and treatment (7th edition) [11] and WHO interim guidance [12], all patients diagnosed with COVID-19 in the considered hospital were enrolled in this study. Clinically diagnosed case was defined as a suspected case with one of the following etiological or serological evidence: (1) positive result of SARS-CoV-2 nucleic acid testing (real-time reverse transcription polymerase chain reaction (RT-PCR) assay); (2) viral gene sequencing that is highly homologous to that of the novel coronavirus; (3) positive result of SARS-CoV-2-specific IgM and IgG antibody, SARS-CoV-2-specific IgG antibody changing from negative to positive, or SARS-CoV-2-specific IgG antibody in the recovery period of COVID-19 four times higher than that in the acute phase. The diagnostic criteria for clinical classification of COVID-19 were referred to the Chinese national guidelines for COVID-19 diagnosis and treatment (7th edition) [11] (Table 1).

\section{Data collection}

Epidemiological and demographic data, symptoms, signs, underlying comorbidities, laboratory findings, chest CT scans, treatment, and outcome data were obtained from patients' medical records. All data were checked by two physicians (Yuhan $\mathrm{Xu}$ and Yilei Guo), and a third researcher ( $\mathrm{Yu} \mathrm{He}$ ) adjudicated any difference in interpretation between the two primary reviewers.

\section{Laboratory procedures}

SARS-CoV-2 in respiratory specimens (throat/nasal swab) was detected via real-time RT-PCR or next-generation sequencing methods. Throat/nasal swab specimens were obtained for SARS-CoV-2 PCR re-examination every other day after remission of clinical symptoms (fever, cough, and dyspnea).

Routine blood examinations (e.g., blood cell counts, blood cell ratio, arterial blood gas analysis, coagulation profile, C-reactive protein, and serum amyloid A), serum biochemical tests (e.g., renal and liver function, creatine kinase, lactate dehydrogenase, procalcitonin, and electrolytes), and chest radiographs or CT scans were conducted for all inpatients.

\section{Evaluation of clinical results}

The clinical characteristics, chest CT images, treatment, and outcomes were compared between the CCVD and non-CCVD groups. The criteria for healing and discharge were referred to the Chinese national guidelines for COVID-19 diagnosis and treatment (7th edition) [11]: (1) absence of fever for at least 3 days; (2) clinical remission of respiratory symptoms and with both lungs showing substantial improvement in chest radiographs or CT scan; (3) two throat/nasal swab samples (obtained at least $24 \mathrm{~h}$ apart) negative for SARS-CoV-2 RNA.

The pneumonia severity index (PSI) is used by medical practitioners to calculate the probability of morbidity and mortality among patients with pneumonia. The evaluation criteria of PSI score and grade were referred to the work of Liu et al. [13]. The higher the PSI total score and grade were, the poorer the condition.

\section{Statistical analysis}

All statistical analyses were performed with SPSS software version 24.0 (IBM Corporation, Chicago, IL, USA). Continuous variables were presented as median (inter-quartile range (IQR)). Categorical variables were presented as $N(\%)$. Comparison between the two groups was analyzed using two-sample Student's $t$-test (normal distribution) or Mann-Whitney $U$ test (abnormal 
Table 1 Diagnostic criteria for clinical classification of COVID-19

\begin{tabular}{ll}
\hline Classification & Diagnostic criteria \\
\hline Mild & Mild clinical symptoms and no evident abnormality \\
& in chest computed tomography $(\mathrm{CT})$ \\
General & With fever, respiratory symptoms, and imaging \\
& characteristics of pneumonia \\
Severe & Compliance with any of the following: \\
& 1. Dyspnea and respiratory rate $\geqslant 30$ per min; \\
& 2. $\mathrm{SPO}_{2} \leqslant 93 \%$ on room air; \\
& 3. $\mathrm{PaO}_{2} / \mathrm{FiO}_{2} \leqslant 300 \mathrm{mmHg}(1 \mathrm{mmHg}=0.133 \mathrm{kPa})$ \\
& Pulmonary imaging showed that the lesions \\
& progressed more than $50 \%$ within $24-48 \mathrm{~h}$ \\
& Compliance with any of the following: \\
& 1. Respiratory failure occurrence and mechanical \\
Critical & ventilation requirement; \\
& 2. Shock; \\
& 3. Combination with other organ failure; intensive \\
& care unit monitoring and treatment requirement
\end{tabular}

distribution) for continuous variables and Chi-square test or Wilcoxon test for categorical variables, where appropriate. A difference with a two-side $\alpha$ less than 0.05 was considered statistically significant.

\section{Results}

\section{Basic clinical and epidemic features of COVID-19 patients on admission}

During the study period, 102 COVID-19 patients, including $46(45.0 \%)$ males, were involved. The patients aged 488 years old and had a median age of 40 years (IQR: $33-$ 56). The most common symptoms upon admission were fever $(72,70.5 \%)$ and cough $(63,61.5 \%)$, followed by fatigue $(24,24.5 \%)$ and expectoration $(22,21.5 \%)$. Six $(5.8 \%)$ patients had no symptoms. A total of $76(74.5 \%)$ patients had a clear epidemiological exposure, 13 (12.7\%) patients denied epidemiological exposure history, and 13 (12.7\%) patients had no clear epidemiological exposure history. In addition, nearly half of the patients had comorbidities. Digestive system diseases $(39,38.2 \%)$ and electrolyte disorders $(39,38.2 \%)$ were the most common comorbidities, followed by CCVDs $(20,19.6 \%)$ and urinary system diseases $(12,11.7 \%)$. In all 102 COVID19 cases, the majority comprised mild and common cases. Four $(3.9 \%)$ cases were severe, and eight $(7.8 \%)$ were PSI grade IV on admission (Table 2).

\section{Comparison of the basic clinical characteristics and epidemic features between CCVD and non-CCVD patients}

Among the 102 COVID-19 patients, 20 (19.6\%) had CCVDs. The median ages of the CCVD and non-CCVD groups were 56 (IQR: 42-62) and 38 years (IQR: 31-51), respectively, showing a statistically significant difference $(P<0.001)$. Moreover, significant differences were observed in the distribution of clinical classification between the two groups $(P=0.012)$. In the study, $15 \%$ of patients with CCVDs developed severe symptoms. By contrast, 1\% developed severe symptoms in the nonCCVD group after admission. In addition, the proportion of PSI grade IV in the CCVD group was significantly higher than that in the non-CCVD group $(P<0.01)$. The results showed that $25 \%$ of patients with CCVD were PSI grade IV, whereas $3.6 \%$ of patients without CCVD were PSI grade IV (Table 2).

\section{Comparison of chest $\mathrm{CT}$ and laboratory results between the two groups}

The findings in CT images can usually reflect the severity of COVID-19. Most patients with COVID-19 had typical imaging features, such as ground-glass opacities (GGO), mixed GGO and consolidation, and nodules (Figs. 1 and 2). However, seven patients in the non-CCVD group had no evident abnormality in the $\mathrm{CT}$ images. The proportion of multiple lobe lesion involvement in the CCVD group was higher than that in the non-CCVD group $(P<0.05$; Table 3).

The distributions of C-reactive protein, fibrinogen, Ddimer, total protein, and serum amyloid A showed a significant difference between the CCVD and non-CCVD groups $(P<0.05)$. We observed that patients with CCVD were more likely to have C-reactive protein $>10 \mathrm{mg} / \mathrm{L}$, fibrinogen $>3.5 \mathrm{~g} / \mathrm{L}$, D-dimer $>1 \mathrm{mg} / \mathrm{L}$, and total protein $<65.0 \mathrm{~g} / \mathrm{L}$. Serum amyloid A in the CCVD group was significantly higher than that in the non-CCVD group $(P<0.05)$. However, the arterial partial $\mathrm{PaO}_{2}$ in the CCVD group was significantly lower than that in the nonCCVD group $(P<0.05$; Table 3$)$.

\section{Comparison of treatments and outcomes between the two groups}

After admission, all patients $(102,100 \%)$ received integrated Chinese and Western medicine treatment. A total of $97(95.1 \%)$ patients received lopinavir and arbidol tablets, $85(83.3 \%)$ patients received interferon, 14 $(13.7 \%)$ patients received immunoglobulin, 9 (8.8\%) patients received antibiotics, $7(6.8 \%)$ patients received corticosteroids, and all patients $(102,100 \%)$ received traditional Chinese medicine (TCM) treatment. After treatment, no mild nor general symptoms of COVID-19 observed during admission became severe, and all patients $(102,100 \%)$ were treated and discharged (Table 4). The median length of stay in the hospital was 16 days (IQR: 11.0-21.3), the median duration of viral shedding was 14 days (IQR: 10.0-20.0) from illness onset, the median time 
Table 2 Clinical and epidemiologic features of COVID-19 on admission

\begin{tabular}{|c|c|c|c|c|}
\hline Variable & Total $(n=102)$ & $\operatorname{CCVD}(n=20)$ & Non-CCVD $(n=82)$ & $P$ value \\
\hline \multicolumn{5}{|l|}{ Age and gender } \\
\hline Age, year (median (IQR)) & $40(33-56)$ & $56(42-62)$ & $38(31-51)$ & $<0.001$ \\
\hline Male $(N(\%))$ & $46(45.0 \%)$ & $8(40 \%)$ & $38(46.3 \%)$ & 0.795 \\
\hline \multicolumn{5}{|l|}{ Vital signs } \\
\hline Respiratory rate, per min & $18(18-19)$ & $18(18-20)$ & $18(18-19)$ & \\
\hline Heart rate, per min & $87(78-100)$ & $86(80-101)$ & $88(76-101)$ & \\
\hline $\begin{array}{l}\text { Systolic blood } \\
\text { pressure }(\mathrm{mmHg})\end{array}$ & $128(115-137)$ & $136(126-144)$ & $125(112-136)$ & \\
\hline Body temperature $\left({ }^{\circ} \mathrm{C}\right)$ & $36.9(36.7-37.5)$ & $36.9(36.7-37.2)$ & $36.9(36.7-37.7)$ & \\
\hline \multicolumn{5}{|l|}{$\begin{array}{l}\text { Signs and symptoms at } \\
\text { admission }(N(\%))\end{array}$} \\
\hline Fever & $72(70.5 \%)$ & $14(70 \%)$ & $58(70.7 \%)$ & 1.000 \\
\hline Cough & $63(61.7 \%)$ & $15(75 \%)$ & $48(58.5 \%)$ & 0.271 \\
\hline Expectoration & $22(21.5 \%)$ & $6(30 \%)$ & $16(19.5 \%)$ & 0.472 \\
\hline Rhinorrhea & $5(4.9 \%)$ & $1(5 \%)$ & $4(4.8 \%)$ & 1.000 \\
\hline Sore throat & $12(11.7 \%)$ & $2(3 \%)$ & $10(12.1 \%)$ & 1.000 \\
\hline Diarrhea & $9(8.8 \%)$ & $3(15 \%)$ & $6(7.3 \%)$ & 0.518 \\
\hline Inappetence & $7(6.8 \%)$ & $3(15 \%)$ & $4(4.8 \%)$ & 0.266 \\
\hline Fatigue & $25(24.5 \%)$ & $3(15 \%)$ & $22(26.8 \%)$ & 0.481 \\
\hline Dizziness and headache & $15(14.7 \%)$ & $3(15 \%)$ & $12(14.6 \%)$ & 1.000 \\
\hline Asymptomatic & $6(5.8 \%)$ & $1(5 \%)$ & $5(6.09 \%)$ & 1.000 \\
\hline Exposure history $(N(\%))$ & & & & 0.333 \\
\hline Yes & $76(74.5 \%)$ & $15(75 \%)$ & $61(74.3 \%)$ & \\
\hline No & $13(12.7 \%)$ & $4(20 \%)$ & $9(10.9)$ & \\
\hline Not clear & $13(12.7 \%)$ & $1(5 \%)$ & $12(14.6 \%)$ & \\
\hline \multicolumn{5}{|l|}{ Comorbidity $(N(\%))$} \\
\hline CCVD & $20(19.6 \%)$ & / & / & \\
\hline Digestive system disease & $39(38.2 \%)$ & $10(50 \%)$ & $29(35.3 \%)$ & 0.342 \\
\hline Urinary system disease & $12(11.7 \%)$ & $5(25 \%)$ & $7(8.5 \%)$ & 0.097 \\
\hline Respiratory system disease & $7(6.8 \%)$ & $0(0 \%)$ & $7(8.5 \%)$ & 0.389 \\
\hline Endocrine system disease & $9(8.8 \%)$ & $4(20 \%)$ & $5(6.0 \%)$ & 0.127 \\
\hline $\begin{array}{l}\text { Chronic hepatitis B virus } \\
\text { infection }\end{array}$ & $10(9.8 \%)$ & $2(10 \%)$ & $8(9.7 \%)$ & 1.000 \\
\hline Electrolyte disorder & $39(38.2 \%)$ & $10(50 \%)$ & $29(35.3 \%)$ & 0.342 \\
\hline Clinical classification $(N(\%))$ & & & & 0.012 \\
\hline Mild & $19(18.6 \%)$ & $2(10 \%)$ & $17(20.7 \%)$ & \\
\hline General & $79(77.5 \%)$ & $15(75 \%)$ & $64(78.0 \%)$ & \\
\hline Severe & $4(3.9 \%)$ & $3(15 \%)$ & $1(1.2 \%)$ & \\
\hline PSI grades & & & & $<0.001$ \\
\hline I & $36(35.2 \%)$ & $0(0 \%)$ & $36(43.9 \%)$ & \\
\hline II & $43(42.1 \%)$ & $8(40 \%)$ & $35(42.6 \%)$ & \\
\hline III & $15(14.7 \%)$ & $7(35 \%)$ & $8(9.7 \%)$ & \\
\hline IV & $8(7.8 \%)$ & $5(25 \%)$ & $3(3.6 \%)$ & $<0.01$ \\
\hline
\end{tabular}

CCVD, cardio-cerebrovascular disease; IQR, interquartile range; PSI, pneumonia severity index.

course of symptom disappearance was 17 days (IQR: 1222), and the median time course of cough disappearance was 20 days (IQR: 14-25.3) (Table 4).

Patients with pre-existing CCVD received more inten- sive integrated treatments to manage their symptoms of COVID-19 than non-CCVD patients. The CCVD group registered a higher need for lopinavir and arbidol tablets (100\% versus $93.9 \%)$, interferon (85\% versus $82.9 \%)$, 

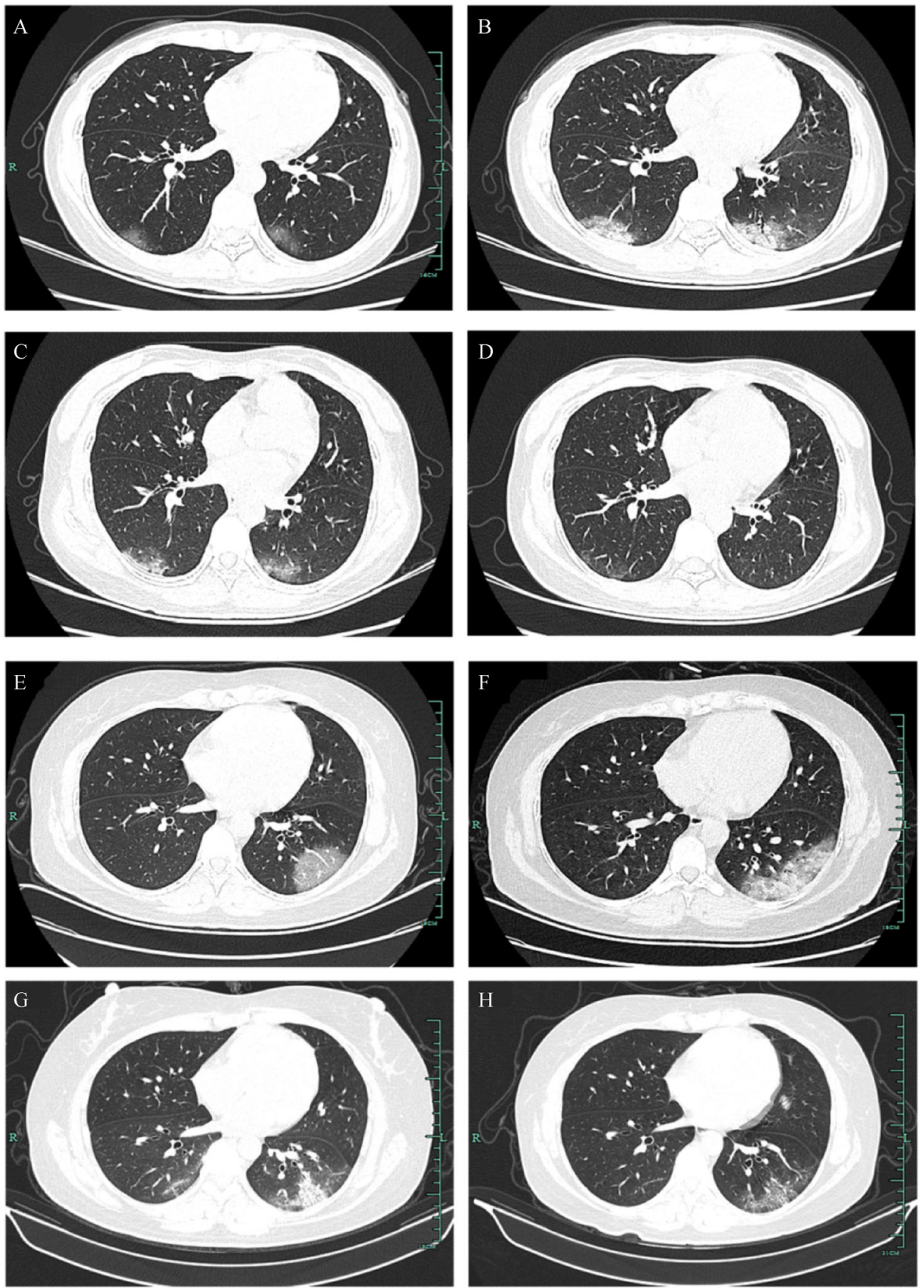

Fig. 1 CT scans of two patients. (A-D) Case 1: (A) Chest CT scans upon admission of a 43-year-old female with CCVD on day 2 after fever, manifested by bilateral multiple GGO. (B) CT scan showing worsening on day 7. The area of subpleural lesions in the lower lobes of both lungs was remarkably enlarged; mixed GGO and consolidation were the main features. (C) Improvements of original lesions on day 12. Lesions in the lower lobes of both lungs reduced in scope and density, presenting as light GGO with patchy consolidations. (D) CT reexamination 14 days after patient's discharge showing that lesions in the lower lobes of both lungs were basically absorbed, presenting as a small light GGO. (E-H) Case 2: (E) Chest CT scans on admission of a 40-year-old female, manifesting multiple GGO in the inferior lobe of the left lung, on day 3 after onset of symptoms. (F) The scope of lesion in the inferior lobe of the left lung was significantly enlarged, with mixed GGO and consolidation on day 7. (G) Improvement of original lesions on day 11; the scope of lesion in the inferior lobe of the left lung was reduced, presenting as light GGO with patchy consolidations. $(\mathrm{H}) \mathrm{CT}$ re-examination showing further absorption of original lesions 7 days after patient's discharge.

antibiotics (15\% versus $7.3 \%)$, and immunoglobin $(20 \%$ versus $12.1 \%$ ) compared with the non-CCVD group. No significant difference was observed in terms of treatment and outcomes in both groups (Table 4). 
Table 3 Chest CT and laboratory results of COVID-19 patients on admission

\begin{tabular}{|c|c|c|c|c|}
\hline Variable & Total $(n=102)$ & $\operatorname{CCVD}(n=20)$ & Non-CCVD $(n=82)$ & $P$ value \\
\hline CT results & & & & 0.042 \\
\hline $\begin{array}{l}\text { No remarkable abnormality } \\
\text { and single lobe lesion }\end{array}$ & $32(31.4 \%)$ & $2(10 \%)$ & $30(36.6 \%)$ & \\
\hline Multiple lobe lesions & $70(68.6 \%)$ & $18(90 \%)$ & $52(63.4 \%)$ & \\
\hline \multicolumn{5}{|l|}{$\begin{array}{l}\text { Laboratory indicators at } \\
\text { admission }\end{array}$} \\
\hline White blood cells $\left(\times 10^{9} / \mathrm{L}\right)$ & $5.24(4.31-7.29)$ & $5.6(4.12-7.37)$ & $5.17(4.48-6.74)$ & 0.696 \\
\hline Lymphocytes $\left(\times 10^{9} / \mathrm{L}\right)$ & $1.24(0.88-1.82)$ & $1.29(0.86-1.87)$ & $1.12(0.85-1.46)$ & 0.067 \\
\hline C-reactive protein $(\mathrm{mg} / \mathrm{L})$ & & & & 0.023 \\
\hline $0-10$ & $70(68.6 \%)$ & $9(45 \%)$ & $61(74.4 \%)$ & \\
\hline$>10$ & $32(31.4 \%)$ & $11(55 \%)$ & $21(25.6 \%)$ & \\
\hline Fibrinogen $(\mathrm{g} / \mathrm{L})$ & & & & 0.014 \\
\hline$\geqslant 1.8$ to $\leqslant 3.5$ & $58(56.9 \%)$ & $6(30 \%)$ & $52(63.4 \%)$ & \\
\hline$>3.5$ & $44(43.1 \%)$ & $14(70 \%)$ & $30(36.6 \%)$ & \\
\hline D-dimer $(\mathrm{mg} / \mathrm{L})$ & & & & 0.017 \\
\hline$\leqslant 0.5$ & $75(73.5 \%)$ & $10(50 \%)$ & $65(79.3 \%)$ & \\
\hline$>0.5$ to $\leqslant 1$ & $8(7.8 \%)$ & $2(10 \%)$ & $6(7.3 \%)$ & \\
\hline$>1$ & $19(18.6 \%)$ & $8(40 \%)$ & $11(13.4 \%)$ & \\
\hline Total protein $(\mathrm{g} / \mathrm{L})$ & & & & 0.034 \\
\hline$<65.0$ & $17(16.7 \%)$ & $7(35 \%)$ & $10(12.2 \%)$ & \\
\hline$\geqslant 65.0$ to $\leqslant 85$ & $85(83.3 \%)$ & $13(65 \%)$ & $72(87.8 \%)$ & \\
\hline AST/ALT & & & & 0.942 \\
\hline$<1$ & $29(28.4 \%)$ & $5(25 \%)$ & $24(29.3 \%)$ & \\
\hline$\geqslant 1$ & $74(72.5 \%)$ & $15(75 \%)$ & $58(70.7 \%)$ & \\
\hline Globulin $(\mathrm{g} / \mathrm{L})$ & $28.6(25.6-32)$ & $30.4(25.3-32.6)$ & $29.3(26.4-32.8)$ & 0.019 \\
\hline Albumin $(\mathrm{g} / \mathrm{L})$ & $40.8(34-43.8)$ & $35(32.3-43.2)$ & $42.6(39.4-45.5)$ & 0.477 \\
\hline Arterial partial $\mathrm{PaO}_{2}(\mathrm{mmHg})$ & $93.9(62.4-113)$ & $79.6(49.4-93.9)$ & $112(92.2-182)$ & 0.028 \\
\hline Oxygen saturation (\%) & $97.8(94.2-98.6)$ & $96.1(83.9-98.0)$ & $99.5(98.5-98.9)$ & 0.386 \\
\hline Serum amyloid A (mg/L) & $55(11-147)$ & $113(28-150)$ & $37(10-122)$ & 0.044 \\
\hline
\end{tabular}

\section{Discussion}

In this study, we observed that COVID-19 patients with pre-existing CCVD presented poorer clinical characteristics, CT image, and laboratory indicators than those without CCVD. The CCVD group showed higher incidences of severe COVID-19, PSI grade IV, and multiple lobe lesions in CT scan compared with the nonCCVD group. Laboratory analysis showed that C-reactive protein, fibrinogen, D-dimer, and serum amyloid A levels were notably higher in the CCVD group compared with the non-CCVD group. The total protein and arterial partial $\mathrm{PaO}_{2}$ levels were lower in the CCVD group compared with the non-CCVD group.

The 102 COVID-19 patients admitted in this study were treated with integrated TCM and Western medicine treatment protocol. After treatment, no mild nor general symptoms of COVID-19 on admission became severe, and no death was recorded. Compared with domestic and foreign data of the same period (as of March 26, 2020,
COVID-19 had caused approximately $4.05 \%$ mortality in China but approximately $4.51 \%$ mortality globally $[14,15])$, our findings reflect the advantages of integrated Chinese and Western medicine treatment. At present, no specific antiviral drugs nor vaccine against COVID-19 infection is available [16]. Western medicine treatment strategies include effective oxygen therapy, antiviral treatment $(\alpha$-interferon atomization inhalation, lopinavir, ritonavir, arbidol, and chloroquine phosphate), antibacterial treatment, etc. Importantly, clinical practice proves that early intervention with TCM can effectively prevent the development of severe symptoms of COVID-19. In TCM, COVID-19 is classified under plague [12]. In this study, our TCM therapeutic principles of COVID-19 were as follows:

(1) Early stage of COVID-19. In this stage, fear of cold, dry cough, dry throat, fatigue, choking sensation in the chest, stomach flatulence, hiccup, loose stool, pale tongue with slimy tongue fur, and floating pulse are usually observed in patients with COVID-19. The basic 
Table 4 Treatment and outcomes

\begin{tabular}{|c|c|c|c|c|}
\hline Variable & Total $(n=102)$ & $\operatorname{CCVD}(n=20)$ & Non-CCVD $(n=82)$ & $P$ value \\
\hline \multicolumn{5}{|l|}{ Treatment } \\
\hline Lopinavir and arbidol tablets & $97(95.1 \%)$ & $20(100 \%)$ & $77(93.9 \%)$ & 0.579 \\
\hline Interferon & $85(83.3 \%)$ & $17(85 \%)$ & $68(82.9 \%)$ & 1.000 \\
\hline $\mathrm{TCM}$ & $102(100 \%)$ & $20(100 \%)$ & $82(100 \%)$ & - \\
\hline Antibiotic & $9(8.8 \%)$ & $3(15 \%)$ & $6(7.3 \%)$ & 0.518 \\
\hline Immunoglobulin & $14(13.7 \%)$ & $4(20 \%)$ & $10(12.1 \%)$ & 0.584 \\
\hline Corticosteroid & $7(6.8 \%)$ & $1(5 \%)$ & $6(7.3 \%)$ & 1.000 \\
\hline \multicolumn{5}{|l|}{ Outcomes } \\
\hline Healed and discharged & $102(100 \%)$ & $20(100 \%)$ & $82(100 \%)$ & - \\
\hline Hospital length of stay, day & $16(11-21.3)$ & $15(11.3-22.8)$ & $16(11-21)$ & 0.918 \\
\hline $\begin{array}{l}\text { Duration of viral shedding } \\
\text { after COVID-19 onset, day }\end{array}$ & $14(10-20)$ & $13.5(9-21.5)$ & $14(10-19)$ & 0.743 \\
\hline $\begin{array}{l}\text { Time course of symptom } \\
\text { disappearance, day }\end{array}$ & $17(12-22)$ & $18.5(12.5-26.3)$ & $16(12-21)$ & 0.412 \\
\hline $\begin{array}{l}\text { Time course of cough } \\
\text { disappearance, day }\end{array}$ & $20(14-25.3)$ & $20.5(14-30.5)$ & $19(14-24)$ & 0.156 \\
\hline
\end{tabular}

prescription is Qingjie Xuantou Feiwei formula: $10 \mathrm{~g}$ honeysuckle (Jinyinhua), $10 \mathrm{~g}$ Polygonum cuspidatum (Huzhang), 10 g Pueraria (Gegen), 6 g Ephedra (Mahuang), $9 \mathrm{~g}$ apricot kernel (Xingren), and $10 \mathrm{~g}$ licorice (Gancao). Based on symptoms, herbs are added or subtracted correspondingly. The usage and dosage are one dose daily; water decoction is $200 \mathrm{~mL}$, twice a day.

(2) Middle stage of COVID-19. Patient in this stage usually have the following symptoms: fever, cough with yellow phlegm, abdominal distension and constipation, chest tightness with shortness of breath, dyspnea, red tongue with yellow greasy tongue fur, and rolling and rapid pulse. Basic prescription: $30 \mathrm{~g}$ gypsum (Shigao), $9 \mathrm{~g}$ raw Ephedra (Shengmahuang), 12 g Lepidium seed (Tinglizi), $10 \mathrm{~g}$ Scutellaria root (Huangqin), $10 \mathrm{~g}$ rawrhubarb (Shengdahuang), 15 g Polygonum cuspidatum (Huzhang), $9 \mathrm{~g}$ apricot kernel (Xingren), $9 \mathrm{~g}$ Areca catechu (Binlang), and $10 \mathrm{~g}$ honeysuckle (Jinyinhua). Herbs are added or subtracted based on symptoms. Usage and dosage are the same as those in the early stage of COVID-19.

(3) Convalescence of COVID-19. In this stage, patients are mostly divided into those with Qi or Qi-yin deficiency syndrome. Qi deficiency syndrome is often accompanied by shortness of breath, fatigue, poor appetite, nausea and vomiting, loose stool, and pale and enlarged tongue with white and greasy tongue fur. Basic prescription of Qi deficiency syndrome: $10 \mathrm{~g}$ Codonopsis pilosula (Dangshen), 15 g Astragalus membranaceus (Zhihuangqi), $9 \mathrm{~g}$ Pinelliae Rhizoma Praeparatum (Fabanxia), $10 \mathrm{~g}$ Dried Tangerine Peel (Chenpi), $9 \mathrm{~g}$ Poria Cocos (Fuling), $10 \mathrm{~g}$ Agastache Rugosus (Huoxiang), 6 g Fructus Amomi (Sharen), and $15 \mathrm{~g}$ Rhizoma Dioscoreae (Shanyao). Qiyin deficiency syndrome is often accompanied by fatigue, dyspnea, thirst, red tongue with little coating, and weak pulse. Basic prescription of Qi-yin deficiency syndrome: 10 g Radix Glehniae (Beishashen), 10 g Radix Pseudostellariae, $10 \mathrm{~g}$ Polygonatum odoratum (Yuzhu), $10 \mathrm{~g}$ Ophiopogon japonicus (Maidong), 12 g Radix Trichosanthis (Tianhuafen), $6 \mathrm{~g}$ Scutellaria root (Huangqin), $9 \mathrm{~g}$ apricot kernel (Xingren), $10 \mathrm{~g}$ honeysuckle (Jinyinhua), 10 g Radix Paeoniae Rubra (Chishao), 10 g Rhizoma Dioscoreae (Shanyao), and 6 g licorice (Gancao). Herbs are added or subtracted based on symptoms. Usage and dosage are the same as those in the early stage of COVID19.

(4) Severe and critical cases of COVID-19. Given that the severe and critical cases of COVID-19 always lead to kidney, liver, and other organ failure and shock and require ICU monitoring [17], patients cannot take Chinese medicine orally. Therefore, Chinese medicine injections, including Xingnaojing, Xuebijing, Danhong (DHI), and Shenmai injections, should be applied.

Given that elderly patients with COVID-19 are usually accompanied by CCVD and other systemic diseases, they are likely to develop severe and critical diseases with high mortality [18]. Studies published on patients admitted to the ICU with COVID-19 show that COVID-19 is linked to coagulopathy and thrombotic risk [8,19-23]. Klok et al. [24] confirmed the extremely high cumulative incidence (31\% of 184 patients) of thrombotic complications (symptomatic acute pulmonary embolism, ischemic stroke, myocardial infarction, systemic arterial embolism, and deep vein thrombosis) in critical COVID-19 patients of three Dutch hospitals, and patients with thrombotic complications had a more than fivefold higher risk of allcause death. Helms et al. [25] conducted a multicenter study of 150 COVID-19 patients and found a $43 \%$ prevalence of thrombosis, which occurred despite 
prophylactic or therapeutic anticoagulation. These pieces of evidence suggest that we should focus on the application of TCM for promoting blood circulation and removing stasis, such as DHI (China Food and Drug Administration approval No. Z20026866). DHI is extracted from Salviae miltiorrhizae Radix and Carthami tinctorii Flos, and it can be used to treat CCVD [26-30].

The present study has several limitations. First, given the retrospective nature of the study, recall biases might have existed in patient signs and symptoms at admission. Second, all data were obtained from Xixi Hospital of Hangzhou, Zhejiang Province, China, which mainly received mild and general cases of COVID-19 patients. Therefore, this study lacks the analysis of severe and critical patients. Third, we did not quantify the viral load of SARS-CoV-2, and the PCR results of upper respiratory samples have the potential to be false negative [19]. Hence, studies on the dynamic changes in viral load are still warranted. Finally, this study is a single-center, smallsample retrospective study and may inadequately reflect the overall complexity of the general population. Therefore, large-scale prospective cohort studies will be required to further gain insights into the clinical features of patients with COVID-19 and pre-existing CCVD.

\section{Acknowledgements}

This work was supported by the National Natural Science Foundation of China (No. 81930111) and Zhejiang Province Highlevel Talents Project (No. 2019R51002).

\section{Compliance with ethics guidelines}

Yu Wang, Lan Li, Yuanjiang Pan, Yu He, Zuhua Chen, Yunhao Xun, Yuhan Xu, Yilei Guo, Jiehong Yang, Jianchun Guo, and Haitong Wan declare no conflict of interest. Given the retrospective nature of this study, informed consent was waived. The study was approved by the Ethics Committee of Xixi Hospital of Hangzhou (Approval No.: 2020-14).

\section{References}

1. Wei X, Li X, Cui J. Evolutionary perspectives on novel coronaviruses identified in pneumonia cases in China. Natl Sci Rev 2020; 7(2): 239-242

2. Wu Z, McGoogan JM. Characteristics of and important lessons from the coronavirus disease 2019 (COVID-19) outbreak in China: summary of a report of 72314 cases from the Chinese Center for Disease Control and Prevention. JAMA 2020; 323(13): 1239

3. Zhu N, Zhang D, Wang W, Li X, Yang B, Song J, Zhao X, Huang B, Shi W, Lu R, Niu P, Zhan F, Ma X, Wang D, Xu W, Wu G, Gao GF, Tan W; China Novel Coronavirus Investigating and Research Team. A novel coronavirus from patients with pneumonia in china, 2019.
N Engl J Med 2020; 382(8): 727-733

4. WHO Director-General/Speeches. WHO Director-General's remarks at the media briefing on $2019-\mathrm{nCoV}$ on 11 February 2020. 2020. https://www.who.int/dg/speeches/detail/who-directorgeneral-s-remarks-at-the-media-briefing-on-2019-ncov-on-11-february-2020 (accessed January 4, 2021)

5. Eurosurveillance Editorial Team. Note from the editors: World Health Organization declares novel coronavirus (2019-nCoV) sixth public health emergency of international concern. Euro Surveill 2020; 25(5): $\mathrm{pii}=200131 \mathrm{e}$

6. World Health Organization. Coronavirus disease (COVID-19) situation report-161. 2020. https://www.who.int/docs/defaultsource/coronaviruse/situation-reports/20200629-covid-19-sitrep161.pdf?sfvrsn $=74$ fde64e_2 (accessed January 4, 2021)

7. Wang F, Zheng S, Zheng C, Sun X. Attaching clinical significance to COVID-19-associated diarrhea. Life Sci 2020; 260: 118312

8. Li JY, You Z, Wang Q, Zhou ZJ, Qiu Y, Luo R, Ge XY. The epidemic of 2019-novel-coronavirus (2019-nCoV) pneumonia and insights for emerging infectious diseases in the future. Microbes Infect 2020; 22(2): 80-85

9. Zhou F, Yu T, Du R, Fan G, Liu Y, Liu Z, Xiang J, Wang Y, Song B, Gu X, Guan L, Wei Y, Li H, Wu X, Xu J, Tu S, Zhang Y, Chen H, Cao B. Clinical course and risk factors for mortality of adult inpatients with COVID-19 in Wuhan, China: a retrospective cohort study. Lancet 2020; 395(10229): 1054-1062

10. Chen J, Qi T, Liu L, Ling Y, Qian Z, Li T, Li F, Xu Q, Zhang Y, Xu S, Song Z, Zeng Y, Shen Y, Shi Y, Zhu T, Lu H. Clinical progression of patients with COVID-19 in Shanghai, China. J Infect 2020; 80(5): e1-e6

11. National Health Commission of the People's Republic of China. Diagnosis and Treatment Protocol for COVID-19 (Trial Version 7). 2020. http://www.nhc.gov.cn/cms-search/xxgk/getManuscriptXxgk.htm? id = 318683 cbfaee4191aee29cd774b19d8d (accessed January 4, 2021)

12. World Health Organization. Clinical management of severe acute respiratory infection when novel coronavirus (2019-nCoV) infection is suspected: interim guidance, 28 January 2020. 2020. https:// apps.who.int/iris/handle/10665/330893 (accessed January 4, 2021)

13. Liu K, Chen Y, Lin R, Han K. Clinical features of COVID-19 in elderly patients: a comparison with young and middle-aged patients. J Infect 2020; 80(6): e14-e18

14. World Health Organization. Laboratory testing for coronavirus disease 2019 in suspected human cases: interim guidance, 2 March 2020. 2020. https://apps.who.int/iris/handle/10665/331329 (accessed January 4, 2021)

15. World Health Organization. Coronavirus disease 2019 (COVID-19): situation report, 63. 2020. https://apps.who.int/iris/handle/10665/ 331607 (accessed January 4, 2021)

16. Ahn DG, Shin HJ, Kim MH, Lee S, Kim HS, Myoung J, Kim BT, Kim SJ. Current status of epidemiology, diagnosis, therapeutics, and vaccines for novel coronavirus disease 2019 (COVID-19). J Microbiol Biotechnol 2020; 30(3): 313-324

17. Li Y, Hu Y, Yu J, Ma T. Retrospective analysis of laboratory testing in 54 patients with severe- or critical-type 2019 novel coronavirus pneumonia. Lab Invest 2020; 100(6): 794-800

18. Zhu L, She ZG, Cheng X, Qin JJ, Zhang XJ, Cai J, Lei F, Wang H, Xie J, Wang W, Li H, Zhang P, Song X, Chen X, Xiang M, Zhang C, 
Bai L, Xiang D, Chen MM, Liu Y, Yan Y, Liu M, Mao W, Zou J, Liu L, Chen G, Luo P, Xiao B, Zhang C, Zhang Z, Lu Z, Wang J, Lu H, Xia X, Wang D, Liao X, Peng G, Ye P, Yang J, Yuan Y, Huang X, Guo J, Zhang BH, Li H. Association of blood glucose control and outcomes in patients with COVID-19 and pre-existing type 2 diabetes. Cell Metab 2020; 31(6): 1068-1077.e3

19. Lake MA. What we know so far: COVID-19 current clinical knowledge and research. Clin Med (Lond) 2020; 20(2): 124-127

20. Guan WJ, Ni ZY, Hu Y, Liang WH, Ou CQ, He JX, Liu L, Shan H, Lei CL, Hui DSC, Du B, Li LJ, Zeng G, Yuen KY, Chen RC, Tang CL, Wang T, Chen PY, Xiang J, Li SY, Wang JL, Liang ZJ, Peng YX, Wei L, Liu Y, Hu YH, Peng P, Wang JM, Liu JY, Chen Z, Li G, Zheng ZJ, Qiu SQ, Luo J, Ye CJ, Zhu SY, Zhong NS; China Medical Treatment Expert Group for Covid-19. Clinical characteristics of coronavirus disease 2019 in China. N Engl J Med 2020; 382 (18): 1708-1720

21. Wang D, Hu B, Hu C, Zhu F, Liu X, Zhang J, Wang B, Xiang H, Cheng Z, Xiong Y, Zhao Y, Li Y, Wang X, Peng Z. Clinical characteristics of 138 hospitalized patients with 2019 novel coronavirus-infected pneumonia in Wuhan, China. JAMA 2020; 323(11): 1061-1069

22. Dolhnikoff M, Duarte-Neto AN, de Almeida Monteiro RA, da Silva LFF, de Oliveira EP, Saldiva PHN, Mauad T, Negri EM. Pathological evidence of pulmonary thrombotic phenomena in severe COVID-19. J Thromb Haemost 2020; 18(6): 1517-1519

23. Thomas W, Varley J, Johnston A, Symington E, Robinson M, Sheares K, Lavinio A, Besser M. Thrombotic complications of patients admitted to intensive care with COVID-19 at a teaching hospital in the United Kingdom. Thromb Res 2020; 191: 76-77

24. Klok FA, Kruip MJHA, van der Meer NJM, Arbous MS, Gommers D, Kant KM, Kaptein FHJ, van Paassen J, Stals MAM, Huisman MV, Endeman H. Confirmation of the high cumulative incidence of thrombotic complications in critically ill ICU patients with COVID-
19: an updated analysis. Thromb Res 2020; 191: 148-150

25. Helms J, Tacquard C, Severac F, Leonard-Lorant I, Ohana M, Delabranche X, Merdji H, Clere-Jehl R, Schenck M, Fagot Gandet F, Fafi-Kremer S, Castelain V, Schneider F, Grunebaum L, AnglésCano E, Sattler L, Mertes PM, Meziani F; CRICS TRIGGERSEP Group (Clinical Research in Intensive Care and Sepsis Trial Group for Global Evaluation and Research in Sepsis). High risk of thrombosis in patients with severe SARS-CoV-2 infection: a multicenter prospective cohort study. Intensive Care Med 2020; 46(6): 1089-1098

26. Wang K, Zhang D, Wu J, Liu S, Zhang X, Zhang B. A comparative study of Danhong injection and Salvia miltiorrhiza injection in the treatment of cerebral infarction: a systematic review and metaanalysis. Medicine (Baltimore) 2017; 96(22): e7079

27. Feng X, Li Y, Wang Y, Li L, Little PJ, Xu SW, Liu S. Danhong injection in cardiovascular and cerebrovascular diseases: pharmacological actions, molecular mechanisms, and therapeutic potential. Pharmacol Res 2019; 139: 62-75

28. Lyu M, Yan CL, Liu HX, Wang TY, Shi XH, Liu JP, Orgah J, Fan GW, Han JH, Wang XY, Zhu Y. Network pharmacology exploration reveals endothelial inflammation as a common mechanism for stroke and coronary artery disease treatment of Danhong injection. Sci Rep 2017; 7(1): 15427

29. Zhao S, Tang Y, Cai H, Liu W, Zhang L, Chen D, Chen B. Treatment of Danhong Injection combined with Naoxintong Capsule in acute coronary syndrome patients undergoing PCI operation: study for a randomized controlled and double-blind trial. Evid Based Complement Alternat Med 2018; 2018: 8485472

30. Zou JB, Zhang XF, Wang J, Wang F, Cheng JX, Yang FY, Song X, Wang Y, Liang YL, Shi YJ. The therapeutic efficacy of Danhong injection combined with percutaneous coronary intervention in acute coronary syndrome: a systematic review and meta-analysis. Front Pharmacol 2018; 9: 550 